\title{
MicroRNA-147 targets BDNF to inhibit cell proliferation, migration and invasion in non-small cell lung cancer
}

\author{
FANG LI, XIANFANG WANG and LINGLING YANG \\ Department of Respiratory Medicine, People's Hospital of Rizhao, Rizhao, Shandong 276500, P.R. China
}

Received July 24, 2019; Accepted January 13, 2020

DOI: 10.3892/ol.2020.11715

\begin{abstract}
Lung cancer is one of the most common cancers that threaten human life and health. Recently, microRNAs (miRNAs) have been shown to play a unique role in many malignancies. Although the dysregulation of miR-147 has been detected in non-small cell lung cancer (NSCLC), the biological function of miR-147 is still unknown in NSCLC. The expression of miR-147 was observed by real-time quantitative polymerase chain reaction (RT-qPCR). Methyl thiazolyl tetrazolium (MTT) and Transwell assays were used to investigate the function of miR-147 in NSCLC. Target genes of miR-147 were verified using dual luciferase reporter assay. Western blot analysis was used to explore the PI3K/AKT pathway. The expression of miR-147 was decreased in NSCLC tissues, which was associated with poor prognosis in NSCLC patients. Furthermore, overexpression of miR-147 inhibited the viability and metastasis of NSCLC cells. In addition, miR-147 inhibited epithelial-mesenchymal transition (EMT) and inactivated the PI3K/AKT pathway in NSCLC. Furthermore, miR-147 directly targets brain-derived neurotrophic factor (BDNF) and negatively regulates BDNF expression in NSCLC. Upregulation of BDNF attenuated the inhibitory effect of miR-147 in NSCLC.In conclusion, miR-147 inhibits cell proliferation, migration and invasion in NSCLC through suppressing BDNF expression.
\end{abstract}

\section{Introduction}

Lung cancer includes non-small cell lung cancer (NSCLC) and small cell lung cancer (SCLC). The difference between NSCLC and SCLC is not defined by size, but by pathology (1). The incidence of NSCLC is higher and more common than SCLC. In addition, patients with NSCLC have a lower degree of malignancy and better treatment outcome (2). Currently, the treatment of NSCLC is based on the clinical stage of

Correspondence to: Dr Fang Li, Department of Respiratory Medicine, People's Hospital of Rizhao, 126 Taian Road, Donggang, Rizhao, Shandong 276500, P.R. China

E-mail: litpgmt3665@163.com

Key words: miR-147, BDNF, PI3K/AKT, non-small cell lung cancer lung cancer. The NSCLC patients in stage I, II, and IIIA are primarily surgically resected. For patients with significant lymphatic metastasis, chemotherapy or radiotherapy can be performed prior to surgery (3). However, the disease is prone to recurrence after surgery, and the prognosis of patients with advanced NSCLC is poor (4). Therefore, NSCLC still faces enormous challenges in diagnosis and treatment.

A large number of studies have shown that microRNAs (miRNAs) are involved in organ development or pathological processes in humans and animals (5). miRNA exerts its effects by binding to the 3'- untranslated region of the target mRNA, thereby inhibiting gene expression or degrading proteins. In various human cancers, the difference in miRNA function depends on the type of cancer or the difference in downstream genes involved (6). At present, the dysregulation of miR-147 has been widely investigated in several cancers. It has been reported that miR-147 was upregulated in gastric cancer and played a carcinogenic role (7). Sui et al (8) found that miR-147 suppressed proliferation and migration of human hepatocellular carcinoma cells by inhibiting homeobox C6 (HOXC6). These studies suggested that miR-147 has tissue specificity in human cancers. In addition, miR-147 was found to be a diagnostic biomarker for human NSCLC (9). However, the biological function and corresponding mechanism of miR-147 remains unclear in NSCLC and needs to be explored. In addition, few studies have shown that miRNAs are involved in NSCLC by regulating phosphoinositide 3-kinase (PI3K)/protein kinase B (AKT) pathway (10). In other cancers, miR-383 suppressed the development of cervical cancer via downregulating poly(ADP-ribose) polymerase-2 (PARP-2) and regulating the PI3K/AKT signaling pathway (11). Furthermore, miR-147 was found to inhibit AKT phosphorylation in HCT116 colon carcinoma cells (12). Therefore, we suspected that miR-147 may regulate the PI3K/AKT pathway in NSCLC cells.

As a member of the neurotrophin family, brain-derived neurotrophic factor (BDNF) is involved in neurotrophins and neuronal differentiation (13). Furthermore, it has been reported that the $\mathrm{PI} 3 \mathrm{~K} / \mathrm{AKT}$ pathway is activated by the BDNF/tyrosine kinase B (TRKB) and BDNF/p75NTR signaling system (14). The function of BDNF has been found in various cancers. For example, BDNF promoted migration and survival of clear cell renal cell carcinoma cells (15). In addition, BDNF was found to promote cell metastasis in human colon cancer (16). In particular, BDNF was associated 
with poor prognosis in NSCLC patients (17). Upregulation of BDNF has been reported to promote proliferation and invasion of lung squamous cell carcinoma cells (18). However, the relationship between miR-147 and BDNF has not been reported in NSCLC.

In this study, we focused on the effects of miR-147 on NSCLC cell viability and metastasis. In addition, how miR-147 regulates BDNF and the PI3K/AKT pathway was also explored in NSCLC. miR-147 may have diagnostic and therapeutic value for NSCLC.

\section{Patients and methods}

Experimental samples. The tissues used in this study were obtained from 79 patients with NSCLC in People's Hospital of Rizhao (Rizhao, China). All NSCLC patients enrolled in this study were treated only with surgery. Participants provided written informed consent and the study was approved by the Institutional Ethics Committee of People's Hospital of Rizhao.

Cell culture and transfection. Human bronchial epithelial cells (16HBE) and A549 NSCLC cells were obtained from the American Type Culture Collection (ATCC). Next, these cells were incubated in RPMI-1640 medium (Gibco; Thermo Fisher Scientific, Inc.) with $10 \%$ fetal bovine serum (FBS). A549 cells were then transfected with miR-147 mimics or inhibitor as well as BDNF vector (Genechem), respectively, using Lipofectamine 2000 (Invitrogen; Thermo Fisher Scientific, Inc.).

Real-time quantitative polymerase chain reaction ( $R T-q P C R)$. Total RNA isolation was performed using TRIzol reagent (Invitrogen; Thermo Fisher Scientific, Inc). The cDNA solution was synthesized using PrimeScript RT reagent (Takara). RT-qPCR was performed on ABI 7500 thermocycler (Applied Biosystems) using SYBR-Green Premix Ex Taq II (Takara). miR-147 or BDNF was normalized by U6 or GAPDH as an endogenous control. Their expression levels were calculated using the $2^{-\Delta \Delta c t}$ method. The primers used were: miR-147 forward, 5'-CCC CTA TCA CGA TTA GCA TTA A-3' and reverse, 5'-CCC AAG CTT TTA TGT GGT TGT TAC TAT GC-3'; U6 forward, 5'-CTC GCT TCG GCA GCA CA-3' and reverse, 5'-AAC GCT TCA CGA ATT TGC GT-3'; GAPDH forward, 5'-GAA GGT GAA GGT CGG AGT C-3' and reverse, 5'-GAA GAT GGT GAT GGG ATT TC-3'; and BDNF forward, 5'-CTA CGA GAC CAA GTG CAA TCC-3' and reverse, 5'-AAT CGC CAG CCA ATT CTC TTT-3'.

Methyl thiazolyl tetrazolium (MTT) assay. First, transfected A549 cells $\left(4 \times 10^{3}\right.$ cells/well) were prepared in 96-well plates. Then, A549 cells were incubated in fresh medium for 24,48 , 72 or $96 \mathrm{~h}$, respectively. Next, $10 \mu \mathrm{l}$ MTT solution was added. The cells were cultured for $4 \mathrm{~h}$. The MTT solution was aspirated and the Formazan solution was added to fully dissolve the crystals. The absorbance at $490 \mathrm{~nm}$ was examined by a microscope (Olympus Corp.).

Transwell assay. Invasion assay was performed in the upper chamber with Matrigel (BD Biosciences). Transfected cells $\left(4 \times 10^{3}\right.$ cells/well) were put in the upper chamber, and lower chamber was filled with $10 \%$ FBS. Next, the cells were fixed and stained. The cell migration assay does not require Matrigel and other procedures are identical to the cell invasion assay. Finally, the migrated and invaded cells were counted using a microscope (Olympus Corp.).

Luciferase reporter assay. First, we constructed a pcDNA3.1 plasmid vector (Promega) containing wild-type or mutant 3'-UTR of BDNF. Next, A549 cells were transfected with the above plasmid and miR-147 mimics and were incubated for $48 \mathrm{~h}$. Finally, luciferase activity was measured using a dual luciferase assay system (Promega).

Western blot analysis. Protein samples were lysed using Radio immunoprecipitation assay (RIPA) lysis buffer (Beyotime). Next, $10 \%$ sodium salt-polyacrylamide gel electrophoresis (SDS-PAGE) protein loading buffer was added to the collected protein samples. After denaturation of the protein, the protein sample was directly loaded into SDS-PAGE gel and transferred into polyvinylidene difluoride (PVDF) membranes. Next, primary antibodies (Vimentin, N-cadherin, E-cadherin, PI3K, AKT, p-PI3K, p-AKT and GAPDH) were added, and the membrane was incubated overnight at $4^{\circ} \mathrm{C}$. The wash solution was added for 5-10 min. Next, the diluted secondary antibody was added and incubated for $1 \mathrm{~h}$ at room temperature. Finally, the protein was examined using an Enhanced chemiluminescence reagent (ECL; Pierce; Thermo Fisher Scientific, Inc.).

Statistical analysis. Data are shown as mean \pm SD and analyzed using Statistical Product and Service Solutions (SPSS) 18.0 (SPSS, Inc.) or GraphPad Prism 6 (GraphPad Systems). Statistical analysis was performed using Chi-square test or one-way analysis of variance (ANOVA) followed by Fisher's least significant difference. The correlation between miR-147 and BDNF expression was examined by Pearson's correlation analysis. The overall survival rates and survival differences were analyzed using the Univariate Kaplan-Meier method followed by log-rank test. $\mathrm{P}<0.05$ was considered to be a statistically significant difference.

\section{Results}

miR-147 expression is decreased in NSCLC tissues. The alteration of miR-147 expression was observed in NSCLC tissues using RT-qPCR. miR-147 expression was decreased in NSCLC tissues compared to normal tissues ( $\mathrm{P}<0.01$; Fig. 1A). Next, the association between miR-147 expression and clinical features in NSCLC tissues was analyzed. It was found that low expression of miR-147 was associated with poor clinical features (including lymph nodes metastasis or tumor stage) in NSCLC patients (Table I). In addition, poor prognosis in NSCLC patients was found to be associated with low miR-147 expression ( $\mathrm{P}=0.042$; Fig. 1B). These results indicate that miR-147 is involved in pathogenesis of NSCLC.

Overexpression of miR-147 suppresses NSCLC cell viability and metastasis. Next, the expression of miR-147 was examined in A549 and 16HBE cell lines. Similarly, downregulation of miR-147 was also assessed in A549 cells compared to 16HBE cells $(\mathrm{P}<0.01$; Fig. 2A). The role of miR-147 in NSCLC was 


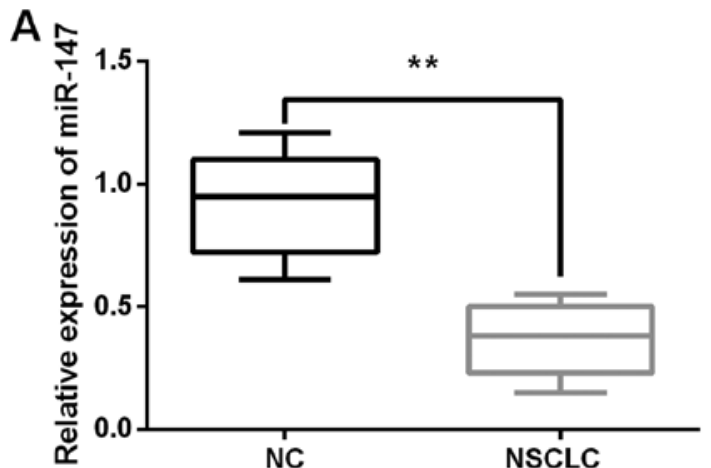

B

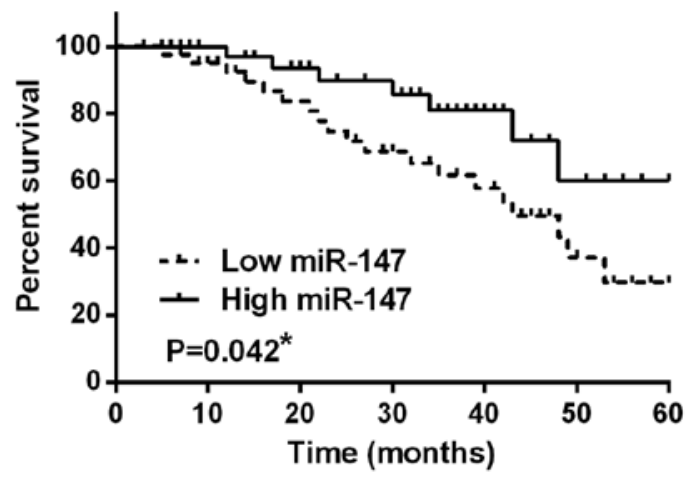

Figure 1. miR-147 expression is decreased in NSCLC tissues. (A) miR-147 expression in NSCLC tissues and normal tissues. (B) Shorter overall survival was detected in NSCLC patients with low miR-147 expression. ${ }^{*} \mathrm{P}<0.05,{ }^{* *} \mathrm{P}<0.01$. NSCLC, non-small cell lung cancer.

Table I. Relationship between miR-147 expression and clinicopathological characteristics of NSCLC patients.

\begin{tabular}{lcccc}
\hline & & \multicolumn{2}{c}{ miR-147 } & \\
Characteristics & Cases & High & Low & P-value \\
\hline Age (years) & & & & 0.06 \\
$\geq 60$ & 44 & 15 & 29 & \\
$\quad<6$ & 35 & 14 & 21 & \\
Sex & & & & 0.13 \\
$\quad$ Male & 43 & 12 & 31 & \\
$\quad$ Female & 36 & 17 & 19 & \\
$\quad \begin{array}{l}\text { Tumor size (mm) } \\
\leq 3\end{array}$ & 52 & 20 & 32 & 0.22 \\
$>3$ & 27 & 9 & 18 & \\
$\quad$ Lymph nodes metastasis & & & & $0.03^{\mathrm{a}}$ \\
$\quad$ Yes & 17 & 5 & 12 & \\
$\quad$ No & 62 & 24 & 38 & \\
$\quad \begin{array}{l}\text { Tumor stage } \\
\text { I-II }\end{array}$ & 57 & 21 & 36 & $0.02^{\mathrm{a}}$ \\
III-IV & 22 & 8 & 14 & \\
\hline
\end{tabular}

Statistical analyses were performed by the $\chi^{2}$ test. ${ }^{\text {a }}<0.05$ was considered statistically significant.

explored in A549 cells with miR-147 mimics or inhibitor. It was found that expression of miR-147 was significantly regulated by miR-147 mimics or inhibitor ( $\mathrm{P}<0.01$; Fig. 2B). MTT assay indicated that overexpression of miR-147 suppressed proliferation of A549 cells ( $\mathrm{P}<0.01$; Fig. 2C). In contrast, downregulation of miR-147 was found to promote cell proliferation $(\mathrm{P}<0.05$; Fig. 2D). In addition, overexpression of miR-147 inhibited cell migration, whereas miR-147 silencing promoted cell migration in A549 cells ( $\mathrm{P}<0.01 ; \mathrm{Fig}$. 2E). Similar results on cell invasion were also detected in A549 cells with miR-147 mimics or inhibitor $(\mathrm{P}<0.01$; Fig. $2 \mathrm{~F})$. Based on these results, it was considered that overexpression of miR-147 suppressed the viability and metastasis of NSCLC cells.

miR-147 blocks epithelial-mesenchymal transition (EMT) and inactivates the PI3K/AKT pathway in NSCLC. To further explain the above conclusion, how miR-147 regulates EMT and the PI3K/AKT pathway was investigated. It was found that miR-147 mimics suppressed the expression of Vimentin and $\mathrm{N}$-cadherin and enhanced E-cadherin expression level in A549 cells (Fig. 3). In contrast, Vimentin and N-cadherin expressions were improved by downregulation of miR-147. E-cadherin expression was suppressed by miR-147 silencing in A549 cells (Fig. 3). Next, it was investigated how miR-147 regulates the PI3K/AKT pathway in A549 cells. Western blot analysis showed that miR-147 mimics downregulated p-PI3K and p-AKT expression in A549 cells (Fig. 3). miR-147 silencing was found to promote expression of p-PI3K and p-AKT. However, miR-147 had no effect on the expression of PI3K and AKT in A549 cells (Fig. 3). These results demonstrate that miR-147 blocks EMT and inactivates the PI3K/AKT pathway in NSCLC.

$B D N F$ is a direct target of miR-147. To further disclose the molecular mechanism of miR-147 in NSCLC, the target gene of miR-147 was searched in the TargetScan (http://www. targetscan.org/) databases. It is predicted that miR-147 has a binding site with the 3'-UTR of BDNF (Fig. 4A). It was found that miR-147 mimics significantly inhibited Wt-BDNF luciferase activity, whereas the luciferase activity of Mut-BDNF was not regulated by miR-147 mimics ( $\mathrm{P}<0.01$; Fig. 4B). Then, BDNF expression was observed in A549 cells containing miR-147 mimics or inhibitor. Overexpression of miR-147 suppressed the expression of BDNF. miR-147 silencing promoted BDNF expression ( $\mathrm{P}<0.01$; Fig. $4 \mathrm{C})$. BDNF expression was detected in NSCLC tissues. RT-qPCR indicated that BDNF was upregulated in NSCLC tissues compared to normal expression $(\mathrm{P}<0.01$; Fig. 4D). Furthermore, a negative correlation between expression of miR-147 and BDNF was identified in NSCLC tissues $\left(\mathrm{P}<0.0001 ; \mathrm{R}^{2}=0.8651\right.$; Fig. 4E). Collectively, miR-147 directly targets BDNF and negatively regulates BDNF expression in NSCLC.

Upregulation of BDNF impairs the inhibitory effect of miR-147 in NSCLC. Finally, BDNF vectors were transfected into A549 cells with miR-147 mimics to explore their interactions. RT-qPCR showed that BDNF vector restored the decreased expression of BDNF induced by miR-147 mimics in A549 cells $(\mathrm{P}<0.01$; Fig. 5A). Furthermore, BDNF vectors 
A

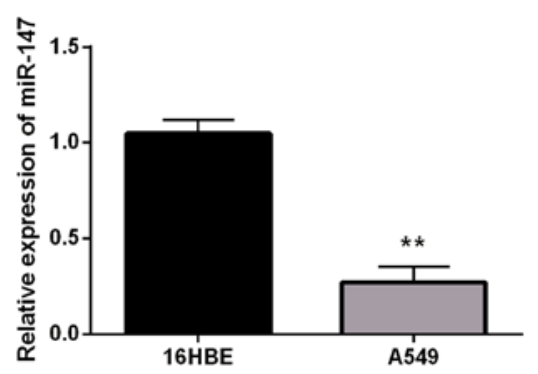

C

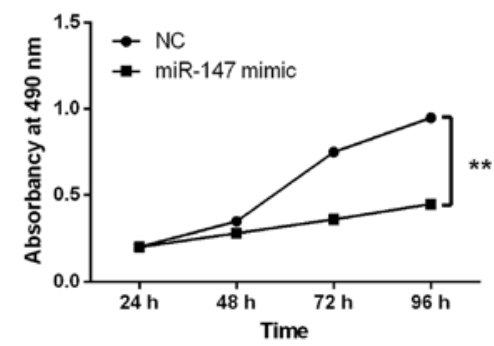

E

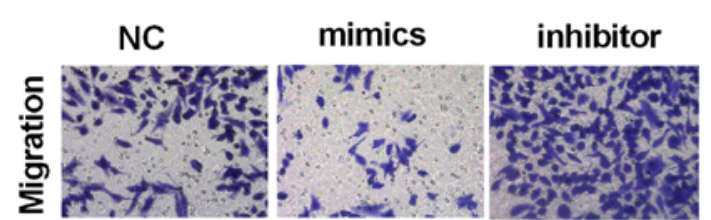

$\mathbf{F}$

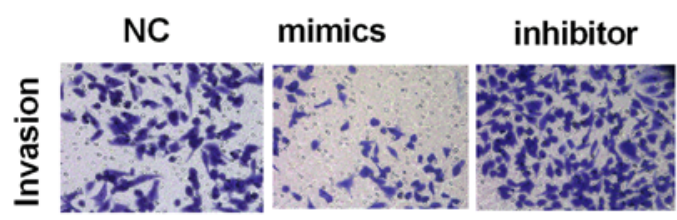

B

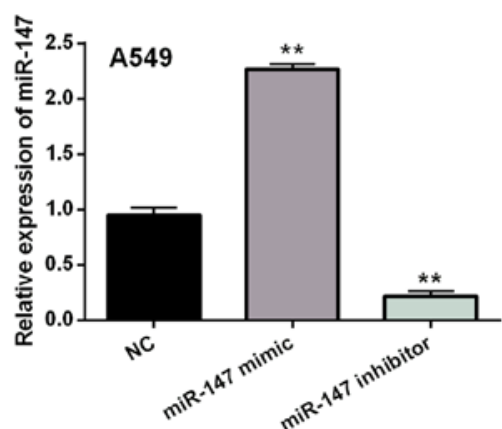

D
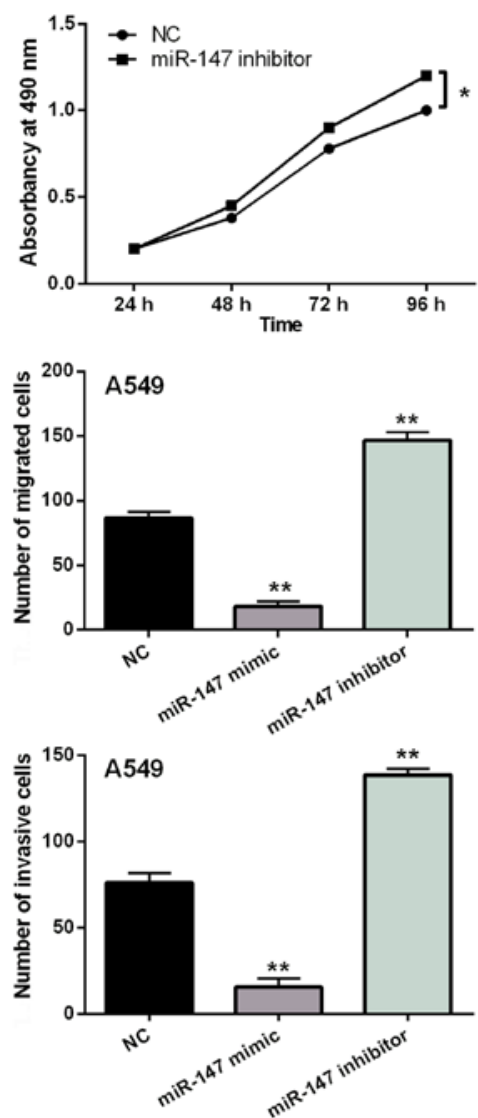

Figure 2. Overexpression of miR-147 suppresses NSCLC cell viability and metastasis. (A) Expression of miR-147 in 16HBE and A549 cells. (B) miR-147 mimics or inhibitor regulated expression in A549 cells. (C-F) Cell proliferation, migration and invasion regulated by miR-147 mimics or inhibitor. ${ }^{*}<0.05$ and ${ }^{* *} \mathrm{P}<0.01$. 16HBE, human bronchial epithelial cells; NSCLC, non-small cell lung cancer.

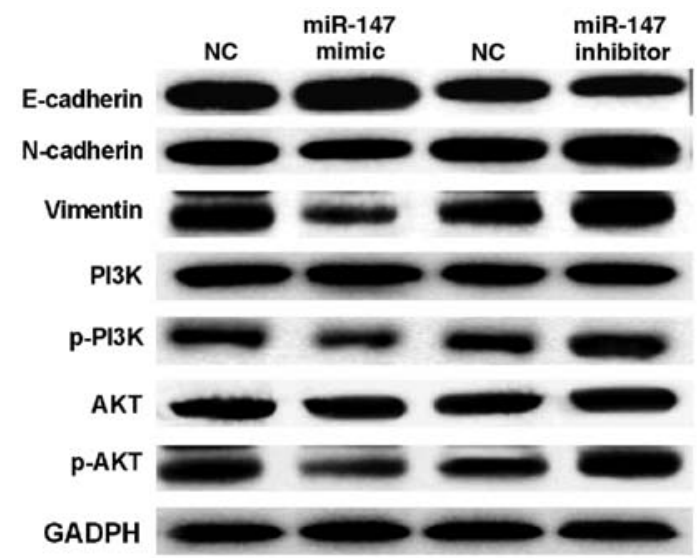

Figure 3. miR-147 blocks EMT and PI3K/AKT pathway in NSCLC. miR-147 regulated protein expression of Vimentin, N-cadherin, E-cadherin, PI3K, AKT, p-PI3K and p-AKT in A549 cells. NSCLC, non-small cell lung cancer; EMT, epithelial-mesenchymal transition. attenuated miR-147-induced inhibition of cell proliferation $(\mathrm{P}<0.01$; Fig. 5B). In addition, BDNF vectors also abolished the inhibitory effect of miR-147 on cell migration and invasion in A549 cells ( $\mathrm{P}<0.01$; Fig. 5C and D). Taken together, upregulation of BDNF attenuated the inhibitory effect of miR-147 in NSCLC.

\section{Discussion}

In this study, it was found that miR-147 expression was decreased in NSCLC tissues, which was associated with poor prognosis in NSCLC patients. In addition, low expression of miR-147 was associated with lymph node metastasis or tumor stage in NSCLC patients. Chu et al (9) also found similar results. However, miR-147 was upregulated in gastric cancer, and downregulation of miR-147 inhibited cell proliferation (19), which is different from the present results. 

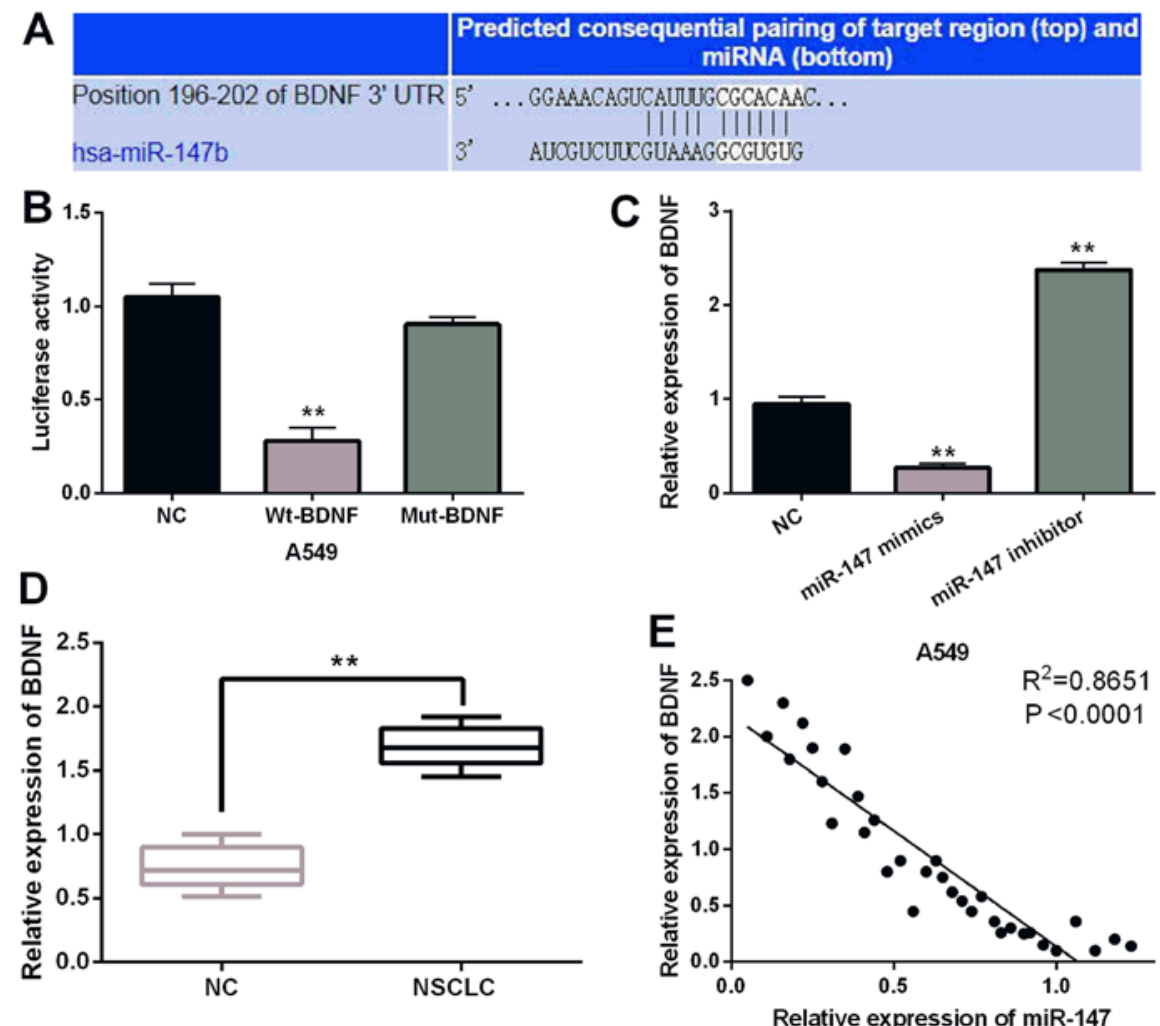

Figure 4. miR-147 directly targets BDNF. (A) The binding sites between miR-147 and BDNF. (B) Luciferase reporter assay. (C) miR-147 mimics or inhibitor regulated BDNF expression in A549 cells. (D) BDNF expression in NSCLC tissues and normal tissues. (E) The negative correlation between miR-147 and BDNF expression in NSCLC tissues, ${ }^{* *} \mathrm{P}<0.01$. BDNF, brain-derived neurotrophic factor; NSCLC, non-small cell lung cancer.

A

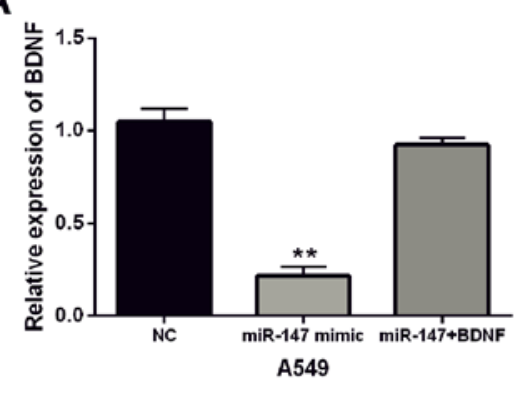

C

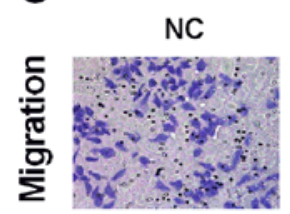

$\operatorname{miR}-147$

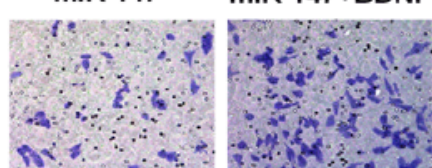

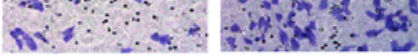

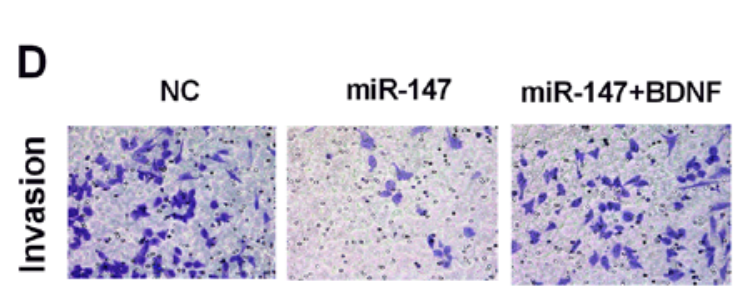

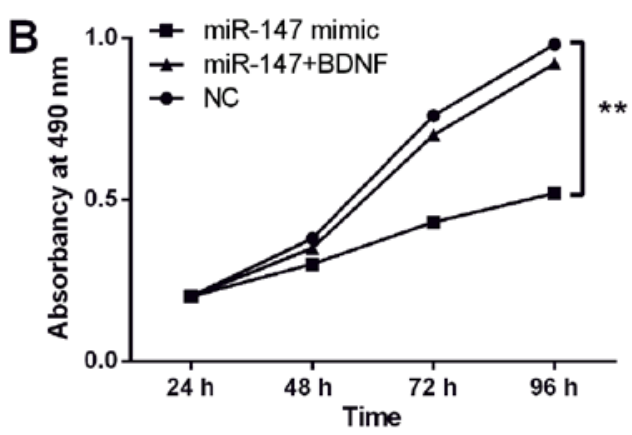
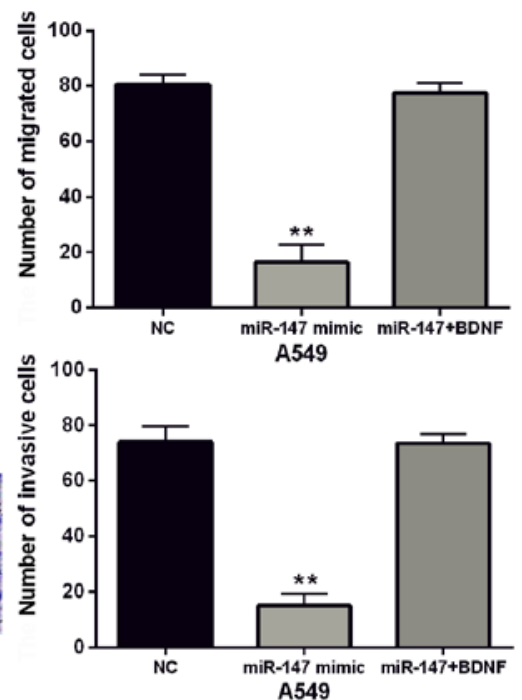

Figure 5. Upregulation of BDNF impairs the inhibitory effect of miR-147 in NSCLC. (A) BDNF vector regulated its expression in A549 cells with miR-147 mimics. (B-D) BDNF vector regulated cell proliferation, migration and invasion in A549 cells with miR-147 mimics. ${ }^{* *} \mathrm{P}<0.01$. BDNF, brain-derived neurotrophic factor; NSCLC, non-small cell lung cancer. 
Upregulation of miR-147 suppressed NSCLC cell viability and metastasis. The difference may be due to the different tumor tissues. Moreover, ectopic expression of miR-147 was found to inhibit epithelial-mesenchymal transition (EMT)-related protein expression in colon cancer cells (20), which is similarly to our results. Our study also found that miR-147 suppressed Vimentin and $\mathrm{N}$-cadherin expression and promoted E-cadherin expression to block EMT in NSCLC. Moreover, miR-147 has been reported to suppress breast cancer cell proliferation, invasion and migration through the PI3K/AKT/mTOR signaling pathway (21). In NSCLC cells, miR-147 was also involved in the PI3K/AKT pathway by suppressing expression of p-PI3K and $\mathrm{p}$-AKT.

EMT and the PI3K/AKT pathway have been reported to be involved in the pathogenesis of NSCLC. For example, miR-149 promoted the expression of E-cadherin in NSCLC (22). Herein, E-cadherin expression was also promoted by miR-147 in NSCLC cells. Furthermore, it has been reported that miR-3188 inhibited NSCLC cell proliferation by regulating p-PI3K and p-AKT expression (23). In addition, PI3K and AKT are key regulators of cell survival, which are critical for cancer development $(24,25)$. Therefore, the expression of PI3K and AKT was detected in A549 cells with miR-147 mimics or inhibitor. It was found that miR-147 suppressed p-PI3K and p-AKT expression to inactivate the PI3K/AKT pathway in NSCLC, which agrees with previous studies. For example, miR-107 has been found to inhibit cell metastasis and tumor growth by inhibiting BDNF and mediating the PI3K/AKT pathway in NSCLC (26). The results suggest that BDNF may be involved in the PI3K/AKT pathway in NSCLC. In the current study, miR-147 was found to directly target BDNF and to negatively regulate BDNF expression in NSCLC. Furthermore, overexpression of miR-147 suppressed p-PI3K and p-AKT expression in NSCLC. Based on these results, we suspect that BDNF can regulate the PI3K/AKT pathway by mediating miR-147. However, this regulatory mechanism is complex. Therefore, further exploration of the regulatory mechanism of miR-147/BDNF/EMT/ PI3K/AKT pathway in NSCLC is required.

Numerous studies have shown that BDNF exerts carcinogenic effects in a variety of cancers. Here, upregulation of BDNF was detected in NSCLC tissues. Similarly, upregulation of BDNF has also been found in breast cancer and colorectal cancer $(27,28)$. As a target gene, BDNF was found to be regulated by several miRNAs, such as miR-15a and miR-613 (29,30). In the present study, miR-147 was also found to negatively regulate BDNF expression in NSCLC. Upregulation of BDNF attenuated the inhibitory effect of miR-147 on cell proliferation, invasion and migration in NSCLC. Similarly, BDNF has been reported to impair the inhibitory effect of miR-497 on cell invasion and tumor growth in thyroid cancer (31). Furthermore, miR-613 inhibited carcinogenesis of gastric cancer by inhibiting BDNF expression (32). miR-147 also inhibited the progression of NSCLC by targeting BDNF.

In conclusion, miR-147 exerts an inhibitory effect in NSCLC. Moreover, miR-147 is involved in tumorigenesis of NSCLC by targeting BDNF or blocking EMT and PI3K/AKT pathway. This study initially indicates that miR-147 may be a promising therapeutic option for NSCLC.

\section{Acknowledgements}

Not applicable.

\section{Funding}

Not funding was received.

\section{Availability of data and materials}

The datasets used and/or analyzed during the present study are available from the corresponding author on reasonable request.

\section{Authors' contributions}

FL designed the study and wrote the manuscript; XW performed the data analyses; LY contributed to the conception of the study. All authors read and approved the final manuscript.

\section{Ethics approval and consent to participate}

The study was approved by the Institutional Ethics Committee of People's Hospital of Rizhao (Rizhao, China). Participants provided written informed consent.

\section{Patient consent for publication}

Not applicable.

\section{Competing interests}

The authors declare that they have no competing interests.

\section{References}

1. Oser MG, Niederst MJ, Sequist LV and Engelman JA: Transformation from non-small-cell lung cancer to small-cell lung cancer: Molecular drivers and cells of origin. Lancet Oncol 16: e165-e172, 2015.

2. Mornex F and Girard N: Gemcitabine and radiation therapy in non-small cell lung cancer: State of the art. Ann Oncol 17: 1743-1747, 2006.

3. Osugi J, Muto S, Matsumura Y, Higuchi M, Suzuki H and Gotoh M: Prognostic impact of the high-sensitivity modified Glasgow prognostic score in patients with resectable non-small cell lung cancer. J Cancer Res Ther 12: 945-951, 2016.

4. Novaes FT, Cataneo DC, Ruiz Junior RL, Defaveri J, Michelin OC and Cataneo AJ: Lung cancer: Histology, staging, treatment and survival. J Bras Pneumol 34: 595-600, 2008.

5. Kloosterman WP and Plasterk RH: The diverse functions of microRNAs in animal development and disease. Dev Cell 11: 441-450, 2006

6. Shenouda SK and Alahari SK: MicroRNA function in cancer: Oncogene or a tumor suppressor? Cancer Metastasis Rev 28: 369-378, 2009.

7. Yao Y, Suo AL, Li ZF, Liu LY, Tian T, Ni L, Zhang WG, Nan KJ, Song TS and Huang C: MicroRNA profiling of human gastric cancer. Mol Med Rep 2: 963-970, 2009.

8. Sui CJ, Xu F, Shen WF, Dai BH, Lu JJ, Zhang MF and Yang JM: MicroRNA-147 suppresses human hepatocellular carcinoma proliferation migration and chemosensitivity by inhibiting HOXC6. Am J Cancer Res 6: 2787-2798, 2016.

9. Chu G, Zhang J and Chen X: Serum level of microRNA-147 as diagnostic biomarker in human non-small cell lung cancer. J Drug Target 24: 613-617, 2016. 
10. Wang Y, Zhao M, Liu J, Sun Z, Ni J and Liu H: miRNA-125b regulates apoptosis of human non-small cell lung cancer via the PI3K/Akt/GSK3 $\beta$ signaling pathway. Oncol Rep 38: 1715-1723, 2017.

11. Teng P, Jiao Y, Hao M and Tang X: microRNA-383 suppresses the PI3K-AKT-MTOR signaling pathway to inhibit development of cervical cancer via down-regulating PARP2. J Cell Biochem 119: 5243-5252, 2018

12. Lee CG, McCarthy S, Gruidl M, Timme C and Yeatman TJ: MicroRNA-147 induces a mesenchymal-to-epithelial transition (MET) and reverses EGFR inhibitor resistance. PLoS One 9: e84597, 2014

13. McAllister AK: Neurotrophins and neuronal differentiation in the central nervous system. Cell Mol Life Sci 58: 1054-1060, 2001.

14. Sandhya VK, Raju R, Verma R, Advani J, Sharma R, Radhakrishnan A, Nanjappa V, Narayana J, Somani BL, Mukherjee KK, et al: A network map of BDNF/TRKB and BDNF/p75NTR signaling system. J Cell Commun Signal 7 : 301-307, 2013.

15. De la Cruz-Morcillo MA, Berger J, Sánchez-Prieto R, Saada S, Naves T, Guillaudeau A, Perraud A, Sindou P, Lacroix A, Descazeaud A, et al: p75 neurotrophin receptor and pro-BDNF promote cell survival and migration in clear cell renal cell carcinoma. Oncotarget 7: 34480-34497, 2016.

16. Huang SM, Lin C, Lin HY, Chiu CM, Fang CW, Liao KF, Chen DR and Yeh WL: Brain-derived neurotrophic factor regulates cell motility in human colon cancer. Endocr Relat Cancer 22: 455-464, 2015.

17. Okamura K, Harada T, Wang S, Ijichi K, Furuyama K, Koga T, Okamoto T, Takayama K, Yano T and Nakanishi Y: Expression of TrkB and BDNF is associated with poor prognosis in non-small cell lung cancer. Lung Cancer 78: 100-106, 2012

18. Zhang SY, Hui LP, Li CY, Gao J, Cui ZS and Qiu XS: More expression of BDNF associates with lung squamous cell carcinoma and is critical to the proliferation and invasion of lung cancer cells. BMC Cancer 16: 171, 2016.

19. Shen J, Niu W, Zhang H, Jun M and Zhang H: Downregulation of microRNA-147 inhibits cell proliferation and increases the chemosensitivity of gastric cancer cells to 5-fluorouracil by directly targeting PTEN. Oncol Res 26: 901-911, 2018.

20. Ning X, Wang C, Zhang M and Wang K: Ectopic expression of miR-147 inhibits stem cell marker and epithelial-mesenchymal transition (EMT)-related protein expression in colon cancer cells. Oncol Res 27: 399-406, 2018.

21. Zhang Y, Zhang HE and Liu Z: MicroRNA-147 suppresses proliferation, invasion and migration through the AKT/mTOR signaling pathway in breast cancer. Oncol Lett 11: 405-410, 2016.
22. Ke Y,Zhao W,Xiong J and Cao R: miR-149 inhibits non-small-cell lung cancer cells EMT by targeting FOXM1. Biochem Res Int 2013: 506731, 2013.

23. Wang C, Liu E, Li W, Cui J and Li T: miR-3188 inhibits non-small cell lung cancer cell proliferation through FOXO1-mediated mTOR-p-PI3K/AKT-c-JUN signaling pathway. Front Pharmacol 9: 1362, 2018.

24. Samuels Y, Wang Z, Bardelli A, Silliman N, Ptak J, Szabo S, Yan H, Gazdar A, Powell SM, Riggins GJ, et al: High frequency of mutations of the PIK3CA gene in human cancers. Science 304 : $554,2004$.

25. Datta SR, Brunet A and Greenberg ME: Cellular survival: A play in three Akts. Genes Dev 13: 2905-2927, 1999.

26. Xia H, Li Y and Lv X: MicroRNA-107 inhibits tumor growth and metastasis by targeting the BDNF-mediated PI3K/AKT pathway in human non-small lung cancer. Int J Oncol 49: 1325-1333, 2016.

27. Kang HJ, Kim JM, Kim SY, Kim SW, Shin IS, Kim HR, Park MH, Shin MG, Yoon JH and Yoon JS: A longitudinal study of BDNF promoter methylation and depression in breast cancer. Psychiatry Investig 12: 523-531, 2015.

28. Tanaka K, Okugawa Y, Toiyama Y, Inoue Y, Saigusa S, Kawamura M, Araki T, Uchida K, Mohri Y and Kusunoki M: Brain-derived neurotrophic factor (BDNF)-induced tropomyosin-related kinase B (Trk B) signaling is a potential therapeutic target for peritoneal carcinomatosis arising from colorectal cancer. PLoS One 9: e96410, 2014.

29. Long J, Jiang C, Liu B, Fang S and Kuang M: MicroRNA-15a-5p suppresses cancer proliferation and division in human hepatocellular carcinoma by targeting BDNF. Tumour Biol 37: 5821-5828, 2016.

30. Li W, Li X, Xin X, Kan PC and Yan Y: MicroRNA-613 regulates the expression of brain-derived neurotrophic factor in Alzheimer's disease. Biosci Trends 10: 372-377, 2016.

31. Wang P, Meng X, Huang Y, Lv Z, Liu J, Wang G, Meng W, Xue S, Zhang Q, Zhang $\mathrm{P}$, et al: MicroRNA-497 inhibits thyroid cancer tumor growth and invasion by suppressing BDNF. Oncotarget 8: 2825-2834, 2017.

32. Ding D, Hou R, Gao Y and Feng Y: miR-613 inhibits gastric cancer progression through repressing brain derived neurotrophic factor. Exp Ther Med 15: 1735-1741, 2018.

This work is licensed under a Creative Commons Attribution-NonCommercial-NoDerivatives 4.0 International (CC BY-NC-ND 4.0) License. 